\title{
On the Non-Symmetric Nash and Kalai-Smorodinsky Bargaining Solutions
}

\author{
Yigal Gerchak*
}

\begin{abstract}
Recently, in some negotiation application areas, the usual assumption that negotiators are symmetric has been relaxed. In particular, weights have been introduced to the Nash Bargaining Solution to reflect the different powers of the players. Yet, we feel that operating with non-symmetric bargaining solutions and their implications is not well understood. We analyze the properties and optimization of the non-symmetric Nash Bargaining Solution and of a non-symmetric Kalai-Smorodinsky Bargaining Solution. We provide extensive comparative statics, then comment on the implications of the concepts in supply chain coordination contexts.
\end{abstract}

Keywords: Nash Bargaining Solution; Non- Symmetric; Kalai-Smorodinsky Bargaining Solution, Supply Chain Coordination

Mathematics Subject Classification: 91A

JEL Classification: C71

Revised: March 5, 2015

\section{INTRODUCTION}

The original Nash Bargaining Solution (NBS) is symmetric in the excesses of the players' utilities over their disagreement utilities. Thus, bargainers are envisioned to be on "equal footing." In an attempt to give one player a "priority" over the other(s), Kalai (1977) axiomatized and presented the non-symmetric NBS (NSNBS). It uses different powers of the excesses over the disagreement values, summing to one. While Kalai (1977) and others explored the axiomatics of the NSNBS, its optimization and economic implications have not been fully explored. As the NSNBS is being recently used in various supply chain settings (Nagarajan and Sosic, 2008; Wu et al., 2009; Mantin et al., 2014), it is important that the SCM and other communities that use such bargaining models will know their general properties. We also consider a lesser-known, non-symmetric version of the Kalai-Smorodinsky solution, where the excesses of the ideal point over the disagreement point are taken to differing powers (Durba, 2001). We then discuss the uses of these concepts in Supply Chain settings.

* Tel Aviv University, Department of Industrial Engineering, Israel, e-mail: yigal@post.tau.ac.il 


\section{NBS ANALYSIS}

The non-symmetric, two-player NBS is the solution of the maximization problem:

$$
\max _{A} f(A) \equiv(A-x)^{\alpha}(K-A-y)^{1-\alpha}, 0 \leq \alpha \leq 1, A \geq x, K \geq x+y,
$$

where $K$ is the quantity of the resource to be divided (the first player receives $A$ ) and $x$ and $y$ are the respective disagreement values. Note that for $A=x$ or $A=K-y, f=0$. The parameter $\alpha$ reflects the relative priority (power) of the first player. When $\alpha=0.5$, one obtains the symmetric NBS. Now:

$$
\partial f / \partial A=(A-x)^{\alpha-1}(K-A-y)^{-\alpha}[\alpha(K-x-y)-(A-x)]
$$

so:

$$
\begin{aligned}
\partial^{2} f / \partial A^{2} & =(A-x)^{\alpha-2}(K-A-y)^{-\alpha-1}\left\{\alpha(\alpha-1)(K-A-y)^{2}\right. \\
& \left.+\alpha^{2}\left((K-x-y)(A-x)-\alpha(A-x)^{2}\right)-\alpha(A-x)(K-A-y)\right\}
\end{aligned}
$$

the sign of which is that of $(\alpha-1)(K-A-y)^{2}-\alpha(A-x)$ which is negative since $0 \leq \alpha \leq 1$.

Thus, the function is concave in $A$.

Now:

$$
\partial f / \partial A=0 \Leftrightarrow \text { the only relevant solution is } A^{*}=\alpha(K-y-x)+x
$$

Note that $A^{*}$ linearly increases in $\alpha$, which is intuitive, as $\alpha$ signifies the power of the first player. It also linearly increases in $x$ and linearly decreases in $y$. When a player has a large "fallback" position, he will have to be "compensated" more.

A more-general scenario is where if one player gets $\mathrm{A}$, the other gets $g(A)$, where $g^{\prime}<0$ and $g^{\prime \prime}<0$. Thus, although what is left for the second player is decreasing in the first's use, that impact is decreasing. So, the objective becomes:

$$
\max _{A} h(A) \equiv(A-x)^{\alpha}[g(A)-y]^{1-\alpha}
$$

Note that $h(x)=h\left(g^{-1}(y)\right)=0$; as with such allocation, both players will rather stay at their disagreement point.

Now:

$$
d h / \partial A=(A-x)^{\alpha-1}[g(A)-y]^{-\alpha}\left\{\alpha[g(A)-y]+(1-\alpha)(A-x) g^{\prime}(A)\right\}
$$

so:

$$
\begin{aligned}
\partial^{2} h / \partial A^{2} & =(\alpha-1)(A-x)^{\alpha-2}[g(A)-y]^{-\alpha-1}\left\{-\alpha g^{\prime}(A)[\alpha(g(A)-y)]\right. \\
& \left.+(1-\alpha)(A-x) g^{\prime}(A)+(g(A)-y)\left[g^{\prime}(A)+A g^{\prime \prime}(A)\right]\right\}
\end{aligned}
$$


Since $0 \leq \alpha \leq 1$, the quantity in \{\} is positive, as $\left(1-\alpha^{2}\right) g^{\prime}(A)[g(A)-y]$ is negative and so is $(1-\alpha) g^{\prime}(A)(A-x)$.

Thus, $h$ is concave in $A$.

The optimality condition is:

$$
\alpha\left[g\left(A^{*}\right)-y\right]+(1-\alpha)\left(A^{*}-x\right) g^{\prime}\left(A^{*}\right)=0
$$

The direction of the dependence of $A^{*}$ on $\alpha$ can be obtained by comparative statics:

$$
d A^{*} / d \alpha=\frac{-g(A)+y+[A-x] g^{\prime}(A)}{(1-\alpha)(A-x) g^{\prime \prime}(A)+g^{\prime}(A)}
$$

As both numerator and denominator are negative, $d A^{*} / d \alpha \geq 0$.

That is, the more powerful the first player, the more he is allocated.

\section{EXAMPLES}

\subsection{EXAMPLE 1}

$$
\begin{gathered}
A^{2}+B^{2}=K \Rightarrow g(A)=\sqrt{K-A^{2}} \\
g^{\prime}(A)=-A / \sqrt{K-A^{2}}<0 \\
g^{\prime \prime}(A)=K / K\left(K-A^{2}\right)^{\frac{3}{2}} \leq 0 \Rightarrow \text { concave } \\
0 \equiv \frac{\partial}{\partial A}=\alpha\left[\sqrt{K-A^{2}}-y\right]+(1-\alpha)(A-x) \cdot \frac{-2 A}{2 \sqrt{K-A^{2}}}
\end{gathered}
$$

so the optimality condition is:

$$
\alpha K-\alpha y \sqrt{K-A^{* 2}}+x(1-\alpha) A^{*}-A^{* 2}=0
$$

That leads to a quartic equation in $A$.

If $x=y=0$, then $A^{*}=\sqrt{\alpha K}$.

If $y=0$

$$
\begin{gathered}
A^{* 2}-x(1-\alpha) A^{*}-\alpha K=0 \\
A^{*}=\frac{x(1-\alpha)+\sqrt{x^{2}(1-\alpha)^{2}+4 \alpha K}}{2}
\end{gathered}
$$




\subsection{EXAMPLE 2}

This example has a convex function $g$.

$$
\begin{gathered}
A B=K \\
A, B \geq 1 \Rightarrow g(A)=K / A \Rightarrow g^{\prime}(A)=-K / A^{2} \Rightarrow g^{\prime \prime}(A)=2 K / A^{3}>0
\end{gathered}
$$

Thus:

$$
\begin{aligned}
& \alpha[K / A-y]+(1-\alpha)(A-x)\left(-K / A^{2}\right)=0 \\
& \Rightarrow A^{*}=\frac{K(2 \alpha-1)+\sqrt{4 \alpha^{2} K^{2}-4 \alpha K^{2}+K^{2}+4 \alpha(1-\alpha) x y K}}{2 \alpha y}
\end{aligned}
$$

Easy to see that $\Delta \geq 0$, and that $A^{*} \geq 0$.

$\frac{d A^{*}}{d \alpha}$ has the sign of

$$
2 K\left[(2 \alpha-1)(K-x y)+\sqrt{4 \alpha^{2} K^{2}-4 \alpha K^{2}+K^{2}-4 \alpha(1-\alpha) x y K}\right]
$$

Thus, if $\alpha<\frac{3-\sqrt{3}}{6} \approx 0.2$, the condition for $\frac{d A^{*}}{d \alpha}>0$ is $K \geq \frac{x y(2 \alpha-1)}{2\left(6 \alpha^{2}-6 \alpha+1\right)}$. If $\alpha>\frac{3-\sqrt{3}}{6}, \frac{d A^{*}}{d \alpha}<0$ always. Interestingly, here an increase in one's power increases its share only if his power was initially low.

\section{NBS WITH $N$ PLAYERS}

Here, the function being maximized is:

$$
\begin{aligned}
& g\left(A_{1, \ldots}, A_{n-1}\right)=\left[\prod_{i=1}^{n-1}\left(A_{i}-x_{i}\right)^{\alpha_{i}}\right]\left(K-\sum_{i=1}^{n-1} A_{i}-x_{n}\right)^{\alpha_{n}} \\
& \alpha_{i} \geq 0, \sum_{i=1}^{n} \alpha_{i}=1 .
\end{aligned}
$$

This model was also discussed by Kalai (1977).

Now:

$$
\begin{aligned}
\frac{\partial g}{\partial A_{i}} & =\alpha_{i}\left(A_{i}-x_{i}\right)^{\alpha_{i}-1} \prod_{j \neq i}\left(A_{j}-x_{j}\right)^{\alpha_{j}}\left(K-\sum_{i=1}^{n-1} A_{i}-x_{n}\right)^{\alpha_{n}} \\
& -\left[\prod_{i=1}^{n-1}\left(A_{i}-x_{i}\right)^{\alpha_{i}}\right] \alpha_{n}\left(K-\sum_{i=1}^{n-1} A_{i}-x_{n}\right)^{\alpha_{n}-1} \\
& =\left[\prod_{j \neq i}^{n-1}\left(A_{j}-x_{j}\right)^{\alpha_{j}}\right]\left(A_{i}-x_{i}\right)^{\alpha_{i}-1}\left(K-\sum_{i=1}^{n-1} A_{i}-x_{n}\right)^{\alpha_{n}-1} \\
& \times\left\{\alpha_{i}\left(A_{i}-x_{i}\right)^{\alpha_{i}-1}\left(K-\sum_{i=1}^{n-1} A_{i}-x_{n}\right)-\alpha_{n}\right\}=0
\end{aligned}
$$


As:

$$
\begin{aligned}
& A_{i}>x_{i} \Rightarrow \\
& \alpha_{i}\left(K-\sum_{i=1}^{n-1} A_{i}-x_{n}\right)\left(A_{i}-x_{i}\right)^{\alpha_{i}-1}=\alpha_{n} . \Rightarrow \\
& A_{i}^{*}=x_{i}+\left[\frac{\alpha_{n}}{\alpha_{i}}\left(K-\sum_{j=1}^{n-1} A_{j}-x_{n}\right]^{\frac{1}{\alpha_{i}-1}}, \quad i=1, \ldots ., n-1\right.
\end{aligned}
$$

Note that, since $\frac{1}{\alpha_{i}-1}<-1, A_{i}^{*}$ decreases in the quantity in [ ]. Thus, $A_{i}^{*} \uparrow x_{i}$, and, $A_{i}^{*} \downarrow K, A_{i} \downarrow \sum A_{j}, A_{i}^{*} \downarrow \alpha_{n}, A_{i}^{*} \uparrow \alpha_{i}$.

The non-intuitive implication is that $A_{i}^{*}$ is decreasing in $K$. We cannot explain this implication.

\section{K-S SOLUTION}

Let $\left(d_{1}, d_{2}\right)$ be the disagreement point and $(M, N)$ the ideal point, where each player obtains his highest utility on $\mathrm{S}$, where $N \geq d_{2}, M \geq d_{1}$. Then, the K-S solution is the pair $\left(u_{1}, u_{2}\right)$, where $u_{1}+u_{2}=S$, satisfying

$$
\frac{u_{2}-d_{2}}{u_{1}-d_{1}}=\frac{N-d_{2}}{M-d_{1}}, u_{1} \geq d_{1}, u_{2} \geq d_{2}
$$

(though the utilities might be negative) (Kalai and Smorodinsky, 1975).

An asymmetric generalization could be (Dubra, 2001):

$$
\frac{u_{2}-d_{2}}{u_{1}-d_{1}}=\frac{\left(N-d_{2}\right)^{\alpha}}{\left(M-d_{1}\right)^{1-\alpha}}, 0 \leq \alpha \leq 1
$$

It follows that:

$$
u_{2}=\frac{d_{2}\left(M-d_{1}\right)^{1-\alpha}+\left(S-d_{1}\right)\left(N-d_{2}\right)^{\alpha}}{\left(M-d_{1}\right)^{1-\alpha}+\left(N-d_{2}\right)^{\alpha}}
$$

while:

$$
u_{1}=S-u_{2}
$$

One can also show that:

$u_{2}^{\prime}(\alpha)=\frac{\left(u_{2}-d_{2}\right)\left(M-d_{1}\right)^{1-\alpha} \log \left(M-d_{1}\right)+\left(S-u_{2}-d_{1}\right)\left(N-d_{2}\right)^{\alpha} \log \left(N-d_{2}\right)}{\left(M-d_{1}\right)^{1-\alpha}+\left(N-d_{2}\right)^{\alpha}}$

So, $u_{2}^{\prime}(\alpha)>0$. This is intuitive.

Note that $u_{1}=S-u_{2}$ is similar to the linear resource constraint $A+B=K$ in NBS. One could thus try the generalization suggested there $\left(u_{1}=g\left(u_{2}, S\right)\right)$, but we shall not do so here. 


\section{EXAMPLE}

Suppose that $u_{1}^{2}+u_{2}^{2}=S$. Note that $d_{i}^{2} \leq S, \quad i=1,2$. The utilities may be negative.

$$
\begin{gathered}
\text { So } \left.\frac{\sqrt{S-u_{1}^{2}}-d_{2}}{u_{1}-d_{1}}=\frac{\left(N-d_{2}\right)^{\alpha}}{\left(M-d_{1}\right)^{1-\alpha}} \equiv R \text { [note that } \frac{d R}{d \alpha}>0\right] \\
\Rightarrow\left(1+R^{2}\right) u_{1}^{2}-2 R\left(R d_{1}-d_{2}\right) u_{1}+d_{1}^{2} R^{2}-S-2 d_{1} d_{2} R+d_{2}^{2}=0 \\
u_{1}=\frac{R\left(R d_{1}-d_{2}\right) \pm \sqrt{S\left(1+R^{2}\right)-\left(R d_{1}-d_{2}\right)^{2}}}{1+R^{2}}
\end{gathered}
$$

To have $\Delta \geq 0$, we require that:

$$
S \geq \frac{\left(R d_{1}-d_{2}\right)^{2}}{1+R^{2}}
$$

We note that, if $N \geq\left(M-d_{1}\right)^{-2}+d_{2}$, then $R^{2} \geq 1$, and it can be shown that then, for the positive roots, $d u_{1} / d R \geq 0$.

\section{POSSIBLE RELEVANCE TO SUPPLY CHAIN ISSUES}

Horizontal supply chains are created when several retailers (at the same echelon of a supply chain that faces random demands) share inventories. This can be achieved either by pooling inventories in a central location or by sharing inventories when one retailer would have a shortage and the other an excess. Such pooling/sharing will result in savings (e.g., Eppen, 1979); but, if the retailers are independent firms, the question is how to divide the savings. This issue was explored using NBS by Hanany and Gerchak (2008). If some retailers are more powerful than others, introducing a-symmetry (NSNBS or NS K-S) would be natural.

Vertical decentralized supply chains [e.g., supplier(s)/manufacturer(s) and retailer(s)] were explored extensively in operations-management literature (e.g., Cachon, 2003). There, after finding a type of contract that will coordinate the supply chain (i.e., make it behave as an integrated chain), the issue that arises is how to divide the profits. Mantin et al. (2014) propose an NSNBS manufacturer-retailer bargaining model. Possibly, the NSK-S solution could also be employed, since each party's ideal point is obtaining all of the profit.

\section{CONCLUDING REMARKS}

The Symmetric NBS is a Non-Transferable Utility (NTU) concept; that is, at that solution, all players' situations improve vis-á-vis their disagreement points. No player needs to compensate another for that to happen (as in TU), which is rather attractive. This property is maintained by the NSNBS, and also holds for the NS K-S solution. We provide some insight into the optimization of these non-symmetric functionals, and extensive comparative statistics for them. 


\section{REFERENCES}

Cachon, G.P., 2003. Supply Chain Coordination with Contracts. Handbooks in Operations Research and Management Science.

Dubra, J., 2001. An Asymmetric Kalai-Smorodinsky Solution. Economics Letters, 73, pp. 131-136.

Eppen, G.D., 1979. Effects of Centralization on Expected Costs in a Multi-Location Newboy Problem. Management Science, 25(5), pp. 498-501.

Hanany, E., Gerchak, Y., 2008. Nash Bargaining Over Allocations in Inventory Pooling Contracts. Naval Research Logistics, 55, pp. 541-550.

Kalai, E., 1977. Non-symmetric Nash Solutions and Replications of a Person Bargaining. International Journal of Game Theory, 6, pp. 129-133.

Kalai, E., Smorodinsky, M., 1975. Other Solutions to Nash's Bargaining Problem. Econometrica, 43, pp. 513-518.

Mantin, B., Krishnan, H., Dhar, T., 2014. The Strategic Role of Third Party Marketplace in Retailing. Production and Operations Management, 23, 1937-1949.

Nagarajan, M., Sosic, G., 2008. Game Theoretic Analysis of Cooperation Supply Chain Agents: Review and Extensions. European Journal of Operational Research, 187, pp. 719-745.

Wu, D., Baron O., Berman, O., 2009. Bargaining in Competitive Supply Chain with Uncertainty. European Journal of Operational Research, 197, pp. 548-558. 